which is not a square; one may add to any set of postulates defining a field these two: $(a)-1$ is a not-square. ( $\beta$ ) The sum of two not-squares is a not-square. On defining $a<b$ to mean that $a-b$ is a not-square, the usual propositions about the symbol < follow at once. If a continuity axiom is added, the system of postulates so obtained defines the real number system. This note is to be published in the Transactions.

22. The third paper by Professor Dickson deals with the generalization of the concept of hypercomplex number systems and of the precise definition of this generalized concept by means of independent postulates. The elements are $a=$ $\left(a_{1}, \cdots, a_{n}\right)$, where the $a_{i}$ are marks of a given field $F$. A system of such elements together with $n^{3}$ fixed marks $\gamma_{i j k}$ form a number system if the following six postulates hold : (1) if $a$ and $b$ are elements of the system then $\left(a_{1}+b_{1}, \cdots\right.$, $\left.a_{n}+b_{n}\right)$ is also an element; (2) the element $0=(0, \cdots, 0)$ occurs in the system ; (3) if 0 occurs, then to any element $a$ corresponds an element $a^{\prime}$ of the system such that $a_{i}+a_{i}^{\prime}=0(i=1$, $\cdots, n) ;(4)$ if $a$ and $b$ are any two elements, then $\left(p_{1}, \cdots, p_{n}\right)$ is an element of the system, where $p_{i}=\sum a_{j} b_{k i} \gamma_{j k i} ;(5)$ the usual relations between the $\gamma$ 's assuring associativity of multiplication ; (6) if $\tau_{1}, \cdots, \tau_{n}$ are marks of $F$ such that $\tau_{1} a_{1}+\cdots+\tau_{n} a_{n}=0$ for every $a$, then $\tau_{1}=0, \cdots, \tau_{n}=0$. It is shown that $n$ units linearly independent with respect to $F$ can be determined. The paper is to appear in the July number of the Transactions.

J. W. Young,

Evanston, ILL., Secretary pro tem. of the Section.

May 20, 1905.

\title{
A GENERAL THEOREM ON ALGEBRAIC NUMBERS.
}

BY PROFESSOR L. E. DICKSON. *

(Read before the American Mathematical Society, December 29, 1904.)

1. LET $r_{1}, \cdots, r_{n}$ belong to a field $F$ and let

$$
\rho^{n}=\sum_{i=1}^{n} r_{i} \rho^{n-i}
$$

* Research assistant to the Carnegie Institution of Washington. 
1905.] GENERAL THEOREM ON ALGEBRAIC NUMBERS. 483

be irreducible in $F$. A multiplication table

$$
\rho^{n-i} \rho^{n-k}=\sum_{s=1}^{n} \gamma_{i k s} \rho^{n-s} \quad(i, k=1, \cdots, n)
$$

enables us to express the product of

in the form $x y=z$,

$$
x=\sum_{i=1}^{n} x_{i} \rho^{n-i}, \quad y=\sum_{k=1}^{n} y_{k} \rho^{n-k} \quad\left(x_{i}, y_{k} \text { in } F\right)
$$

$$
z=\sum_{s=1}^{n} z_{s} \rho^{n-s}, \quad z_{s} \equiv \sum_{i, k}^{1, \ldots, n} \gamma_{i k s} x_{i} y_{k} \quad(s=1, \ldots, n) .
$$

Theorem. Each of the $3 n$ determinants*

$$
C_{i} \equiv\left|\gamma_{i k s}\right|, \quad C_{k}^{\prime} \equiv\left|\gamma_{i k s}\right|, \quad C_{s}^{\prime \prime}=\left|\gamma_{i k s}\right|
$$

is not zero. We have the evaluations

$$
\begin{gathered}
C_{i}=(-1)^{i(n-i)} r_{n}{ }^{n-i}, \quad C_{k}^{\prime}=(-1)^{k(n-k)} r_{n}^{n-k}, \\
C_{1}^{\prime \prime}=\theta_{n}, \quad C_{n}^{\prime \prime}=\theta_{n-1} r_{n}^{n-1},
\end{gathered}
$$

where $\theta_{m}$ is +1 if $m$ is of the form $4 l$ or $4 l+1$, but $\theta_{m}$ is -1 if $m$ is of the form $4 l+2$ or $4 l+3$; while for $1<s<n, C_{s}^{\prime \prime} i s$, aside from sign, the resultant of the equations

$$
\rho^{s-1}=\sum_{i=1}^{s-1} r_{i} \rho^{s-1-i}, \quad 0=\sum_{i=s}^{n} r_{i} \rho^{n-i},
$$

obtained by splitting equation (1) into two parts.

2. That $C_{i} \neq 0$ and $C_{k}^{\prime} \neq 0$ follows readily from the theory of higher complex numbers. For any $y$ and $z$ in $F(\rho), y \neq 0$, there is an unique solution $x$ of $x y=z$; for any $x$ and $z$ in $F(\rho), x \neq 0$, there is an unique solution $y$ of $x y=z$. Hence of the determinants

$$
\Delta \equiv\left|\sum_{i=1}^{n} \gamma_{i k s} x_{i}\right|_{k, s=1, \ldots, n}, \quad \Delta^{\prime} \equiv\left|\sum_{k=1}^{n} \gamma_{i k s} y_{k l}\right|_{i, s=1, \ldots, n},
$$

$\triangle$ vanishes only if $x_{1}=\cdots=x_{n}=0, \Delta^{\prime}$ vanishes only if $y_{1}=\cdots=y_{n}=0$. But $C_{i}$ is the coefficient of $x_{i}^{n}$ in $\Delta, C_{k}^{\prime}$ is the coefficient of $y_{k}^{n}$ in $\Delta^{\prime}$.

3. From (1) we obtain

$$
\rho^{n+j}=\sum_{s=1}^{n} b_{s j} \rho^{n-s}, \quad b_{s j} \equiv \sum_{i=0}^{j} r_{s+j-i} f_{i},
$$

\footnotetext{
* Sections of the cubic array $\left|\gamma_{i k s}\right|$ by planes parallel to the faces.
} 
484 general theorem on algebraic numbers. [June, where $r_{t}(t>n)$ is to be replaced by zero, and where

$$
\begin{aligned}
& f_{0}=1, \quad f_{1}=r_{1}, \quad f_{2}=r_{2}+r_{1}^{2}, \quad f_{3}=r_{3}+2 r_{2} r_{1}+r_{1}^{3}, \\
& f_{4}=r_{4}+2 r_{3} r_{1}+r_{2}^{2}+3 r_{2} r_{1}^{2}+r_{1}^{4}, \\
& f_{5}=r_{5}+2 r_{4} r_{1}+2 r_{3} r_{2}+3 r_{3} r_{1}^{2}+3 r_{2}^{2} r_{1}+4 r_{2} r_{1}^{3}+r_{1}^{5},
\end{aligned}
$$

$f_{i}$ being the sum of all terms of weight $i$ in the $r$ 's with the num. ber of arrangements of the $r$ 's as coefficients. Then

$$
f_{j+1}=\sum_{i=0}^{j} r_{1+j-i} f_{i}, f_{j+1}=b_{1 j}
$$

A comparison of $(8)_{1}$ with $(2)$ gives

$$
\gamma_{i k s}=b_{s n-i-k} \quad(k \leqq n-i), \quad \gamma_{i k s}=\delta_{k n-i+s}(k>n-i),
$$
where

$$
\delta_{k l k}=1, \delta_{k t}=0 \text { if } t \neq k \text {. }
$$

4. Applying (10) for the first $n-i$ rows and $(10)_{2}$ for the last $i$ rows, we obtain for $C_{i}=\left|\gamma_{i k s}\right|$ the expression

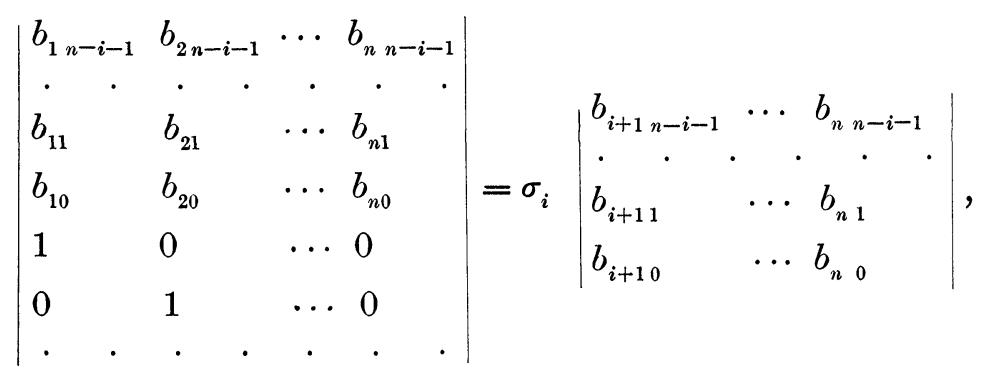

where $\sigma_{i}=(-1)^{i(n-i)}$. Now by $(8)_{2}, b_{s 0}=r_{s}, b_{s 1}-f_{1} r_{s}=r_{s+1}$, $b_{s 2}-f_{1} r_{s\urcorner \cdot 1}-f_{2} r_{s}=r_{s+2}, \cdots$ Thus,

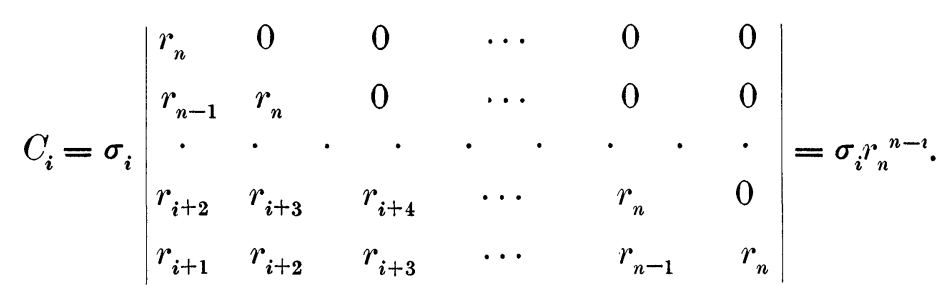

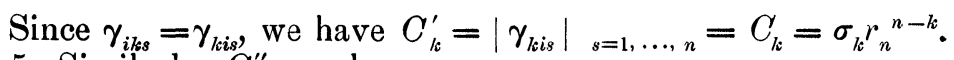
5. Similarly, $C_{s}^{\prime \prime}$ equals 
1905.] GENERAL THEOREM ON ALGEBRAIC NUMBERS. 485

$$
\left|\begin{array}{llllllllll}
b_{s n-2} & b_{s n-3} & b_{s n-4} & . & . & . & . & b_{s 1} & b_{s 0} & 0 \\
b_{s n-3} & b_{s n-4} & \cdot & . & . & . & b_{s 1} & b_{s 0} & 0 & 0 \\
b_{s n-4} & \cdot & . & . & . & b_{s 1} & b_{s 0} & 0 & 0 & . \\
\cdot & . & . & . & \cdot & . & . & . & . & . \\
\cdot & . & . & b_{s 1} & b_{s 0} & 0 & 0 & . & 0 & 1 \\
. & . & b_{s 1} & b_{s 0} & 0 & 0 & . & 0 & 1 & 0 \\
. & . & . & . & . & . & . & . & . & . \\
b_{s 1} & b_{s 0} & 0 & 0 & . & 0 & 1 & 0 & . & . \\
b_{s 0} & 0 & 0 & . & 0 & 1 & 0 & . & . & 0 \\
0 & 0 & . & 0 & 1 & 0 & . & . & 0 & 0
\end{array}\right|
$$

All the elements in a parallel to the left-hand diagonal are equal. The parallel with the elements 1 joins the sth element of the last row with the sth element of the last column.

In $C_{1}^{\prime \prime}$ the elements of the left-hand diagonal are all 1 , and every element below this diagonal is zero. Hence $C_{1}^{\prime \prime}=\theta_{n}$ $= \pm 1$. In $C_{n}^{\prime \prime}$ every element in and below the left-hand diagonal is zero except the element 1 at the intersection of the last row and last column. Hence $C_{n}^{\prime \prime}=\theta_{n-1} b_{n 0}^{n-1}$.

For $1<s<n, C_{s}^{\prime \prime}=(-1)^{n-s} D_{s n}$, where $D_{s n}$ is the minor of the element 1 in the last column. We proceed to the proof that $D_{s n}$ is the eliminant by Sylvester's dialytic method of equations (7). The nature of the general proof will be clear from the following two illustrations:

For $n=5, s=2, D_{s n}$ is

$$
\left|\begin{array}{llll|l}
b_{23} & b_{22} & b_{21} & b_{20} \\
b_{21} & b_{20} & 0 & 1 \\
b_{20} & 0 & 1 & 0 \\
0 & 1 & 0 & 0
\end{array}\right| \begin{aligned}
& b_{20}=r_{2}, \\
& b_{21}=r_{3}+r_{2} f_{1}, f_{1}=r_{1}, \\
& b_{22}=r_{4}+r_{3} f_{1}+r_{2} f_{2}, \\
& b_{23}=r_{5}+r_{4} f_{1}+r_{3} f_{2}+r_{2} f_{3} .
\end{aligned} \text {. }
$$

Multiply the fourth column by $-f_{1},-f_{2}^{\prime},-f_{3}$ and add to the third, second, first columns, respectively. In the resulting determinant, multiply the third column by $-f_{1}$ and $-f_{2}$, and add to the second and first columns, respectively. The first and second elements of the second row are now $(\S 3)$ 
DEFORMATION OF SURFACES OF TRANSLATION. [June,

$$
r_{3}+r_{2} r_{1}-f_{3}+f_{2} r_{1}=0, r_{2}-f_{2}+r_{1}^{2}=0 .
$$

Multiply the new second column by $-f_{1}$ and add to the first. There results

$$
D_{25}=\left|\begin{array}{rrrr}
r_{5} & r_{4} & r_{3} & r_{2} \\
0 & 0 & -r_{1} & 1 \\
0 & -r_{1} & 1 & 0 \\
-r_{1} & 1 & 0 & 0
\end{array}\right| \text {, }
$$

the eliminant of $r_{5}+r_{4} \rho+r_{3} \rho^{2}+r_{2} \rho^{3}=0,-r_{1}+\rho=0$.

For $n=4, s=3, D_{s n}$ is

$$
\left|\begin{array}{lll}
b_{32} & b_{31} & b_{30} \\
b_{31} & b_{30} & 0 \\
0 & 0 & 1
\end{array}\right|, \quad \begin{aligned}
& b_{30}=r_{31} \\
& b_{32}=r_{4}+r_{3} f_{1} \\
& b_{32}=r_{4} f_{1}+r_{3} f_{2}
\end{aligned}
$$

the term $r_{5}$ in $b_{32}$ being dropped since $5>n(\S 3)$. Multiply the third column by $-f_{1}$ and $-f_{2}$ and add to the second and first columns, respectively. Multiply the new second column by $-f_{1}$ and add to the first. There results

$$
D_{34}=\left|\begin{array}{rrr}
0 & r_{4} & r_{3} \\
r_{4} & r_{3} & 0 \\
-r_{2} & -r_{1} & 1
\end{array}\right|
$$

the eliminant of $r_{4}+r_{3} \rho=0,-r_{2}-r_{1} \rho+\rho^{2}=0$.

Chicago, December 8, 1904.

\title{
ON THE DEFORMATION OF SURFACES OF TRANSLATION.
}

\author{
BY DR. L. P. EISENHART.
}

(Read before the American Mathematical Society, February 25, 1905.)

Is the January number of the Bulletis* Dr. Burke Smith states the following theorem : The only non-developable surfaces of translation which may be deformed so that their gener-

* "On the deformation of surfaces of translation," p. 187. 